Supporting Information (SI)

\title{
$\mathrm{A} \mathrm{New} \mathrm{Hg}^{2+}$ and $\mathrm{Cu}^{2+}$ Selective Chromo- and Fluoroionophore Based on a Bichromophoric azine.
}

\author{
Rosario Martínez, Arturo Espinosa, Alberto Tárraga, * and Pedro Molina * \\ Departamento de Química Orgánica. Facultad de Química. Universidad de Murcia. \\ Campus de Espinardo, E-30100 Murcia, Spain. \\ E-mail:pmolina@um.es
}

$\underline{\text { Table of contents }}$

$\begin{array}{ll}\text { General comments } & \text { S2 }\end{array}$

$\begin{array}{ll}\text { Computational details } & \text { S2 }\end{array}$

$\begin{array}{ll}\text { Relevant spectral and characterization data of compound } 1 & \text { S3 }\end{array}$

Figure SI 1. UV/Vis spectra obtained during the titration of 1 in $\mathrm{CH}_{3} \mathrm{CN}$ $\left(\mathrm{c}=2.5 \times 10^{-5} \mathrm{~mol} \mathrm{dm}^{-3}\right)$ with $\mathrm{Cu}(\mathrm{OTf}) \quad \mathrm{S} 4$

Figure SI 2. Excitation spectra of $\mathbf{1}+1$ equiv. of $\mathrm{Hg}\left(\mathrm{ClO}_{4}\right)_{2} \quad \mathrm{~S} 4$

Figure SI 3. Semilogarithmic plot for determining the detection limit for $\mathrm{Hg}^{2+} \mathrm{S} 5$

Figure SI 4. Excitation spectra of $1+1$ equiv. of $\mathrm{Cu}(\mathrm{OTf})_{2} \quad$ S5

Figure SI 5. Semilogarithmic plot for determining the detection limit for $\mathrm{Cu}^{2+} \mathrm{S} 6$

Figure SI 6. Comparison of the fluorescence spectra obtained after addition of 1 equiv of $\mathrm{Hg}\left(\mathrm{ClO}_{4}\right)_{2}$ or $\mathrm{Cu}(\mathrm{OTf})_{2}$ to a solution of 1

Figure SI 7. Plot showing the selectivity of $\mathbf{1}$ to different metal ions S8

Figure SI 8. Calculated structure for the $(\mathbf{1} \cdot \mathrm{Cu})_{2}{ }^{4+}$ complex $\quad S 9$ 
Cartesian coordinates for the calculated structure of the $(\mathbf{1} \cdot \mathrm{Hg})_{2}{ }^{4+}$ complex

\section{General comments.-}

Melting points were determined on a hot-plate melting point apparatus and are uncorrected. IR spectra were determined as Nujol emulsions or films. ${ }^{1} \mathrm{H}$ - and ${ }^{13} \mathrm{C}-\mathrm{NMR}$ spectra were recorded at $300 \mathrm{MHz}$. Chemical shifts refer to signals of tetramethylsilane in the case of ${ }^{1} \mathrm{H}$ and ${ }^{13} \mathrm{C}$ spectra.

Quantum yield values were measured with respect to anthracene as standard $(\Phi=$ $0.27 \pm 0.03),{ }^{1}$ using the equation $\Phi_{\mathrm{x}} / \Phi_{\mathrm{s}}=\left(\mathrm{S}_{\mathrm{x}} / \mathrm{S}_{\mathrm{s}}\right)\left[\left(1-10^{-\mathrm{As}}\right) /\left(1-10^{-\mathrm{Ax}}\right)\right],{ }^{2}$ where $\mathrm{x}$ and $\mathrm{s}$ indicate the unknown and standard solution, respectively, $\Phi$ is the quantum yield, $S$ is the area under the emission curve and $\mathrm{A}$ is the absorbance at the excitation wavelength $\left(\lambda_{\text {exc }}=350 \mathrm{~nm}\right)$.

\section{Computational details.-}

Calculated geometries were initially optimized with the CAChe ${ }^{3}$ program without symmetry constraints at the semiempirical PM5 level, which has been reported to give accurate results comparable with ab initio HF or DFT calculations. ${ }^{4}$ The obtained structures were then fully refined with tight convergence criteria at the final DFT level with the Gaussian 03 package, ${ }^{5}$ using the $\mathrm{B}^{2} \mathrm{LYP}^{6}$ functional and the $6-31 \mathrm{G}^{*}$ basis set for all atoms excepting mercury for which the Stuttgart relativistic small-core basis set ${ }^{7}$ $((8 s 8 p 7 d) \rightarrow[6 s 6 p 4 d]$ contraction $)$ with effective core potential was used. Bond orders were characterized by the Wiberg's bond index ${ }^{8}$ (WBI) and calculated at the aforementioned DFT level with the Natural Bond Orbital (NBO) method as the sum of squares of the off-diagonal density matrix elements between atoms, as formulated in terms of the natural atomic orbital (NAO) basis set.

Relevant spectral and characterization data of compound 1. Yield: 65\%; $\operatorname{mp}>300^{\circ} \mathrm{C}$. IR (Nujol) $v_{\max }\left(\mathrm{cm}^{-1}\right): 1602,1184,847,821,748,713 .{ }^{1} \mathrm{H}$ NMR $\left(\mathrm{CDCl}_{3}\right): \delta=8.14(\mathrm{t}, 2 \mathrm{H}, \mathrm{J}=7.6 \mathrm{~Hz}), 8.25(\mathrm{~d}, 2 \mathrm{H}, \mathrm{J}=8.8 \mathrm{~Hz}), 8.31(\mathrm{~d}, 2 \mathrm{H}, \mathrm{J}=8.8 \mathrm{~Hz})$, 
8.38-8.42 (m, 8H), 8.78 (d, 2H, J=8.0 Hz), 9.22 (d, 2H, J=9.3Hz), 9.79 (s, 2H). ${ }^{13} \mathrm{C}$ NMR $\left(\mathrm{CDCl}_{3}\right): \delta=122.8(2 \times C H), 123.8\left(2 \times C_{q}\right), 124.4(2 \times C H), 125.4(2 \times C H), 125.6$ $(2 \times C H), 125.9(2 \times C H), 126.2\left(2 x_{q}\right), 126.4(2 \times C H), 126.6\left(2 x_{q}\right), 126.7(2 \times C H)$, $128.3(2 \times C H), 128.4(2 \times C H), 129.3\left(2 \times C_{q}\right), 129.9\left(2 \times C_{q}\right), 130.4\left(2 \times C_{q}\right), 132.4\left(2 \times C_{q}\right)$, $159.3(2 \times \mathrm{CH}=\mathrm{N})$. MS (EI): $\mathrm{m} / \mathrm{z}(\%): 456\left(\mathrm{M}^{+}, 59\right), 227(100), 201(92)$. Anal Calc for $\mathrm{C}_{34} \mathrm{H}_{20} \mathrm{~N}_{2}: \mathrm{C}, 89.45 ; \mathrm{H}, 4.42 ; \mathrm{N}, 6.14$. Found: $\mathrm{C}, 89.20 ; \mathrm{H}, 4.30 \mathrm{~N}, 6.28$. 


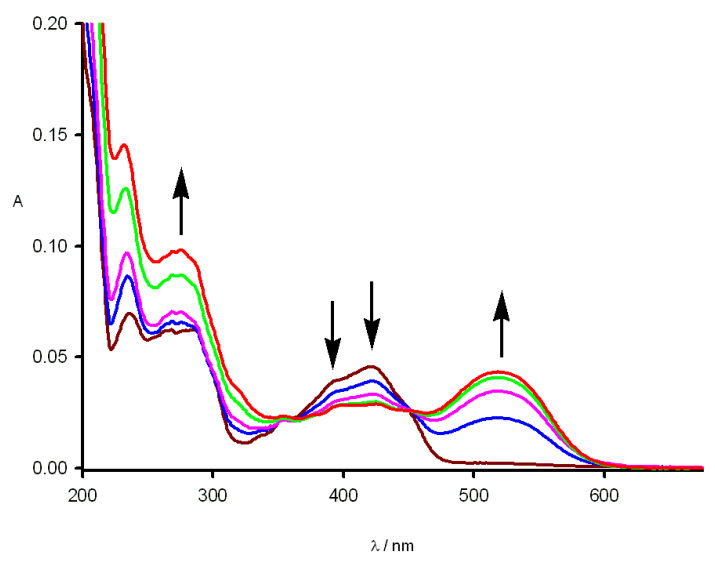

Figure SI 1. UV/Vis spectra obtained during the titration of 1 in $\mathrm{CH}_{3} \mathrm{CN}\left(\mathrm{c}=2.5 \times 10^{-5}\right.$ mol dm${ }^{-3}$ ) with $\mathrm{Cu}(\mathrm{OTf})_{2}$. The initial spectrum (black) is that of starting $\mathbf{1}$ and the final spectrum (red) corresponds to the complex form $\mathbf{1} \cdot \mathrm{Cu}^{2+}$ after addition of 1 equiv. of $\mathrm{Cu}^{2+}$. Arrows indicate the absorptions that increased (up) and decreased (down) during the titration experiments.

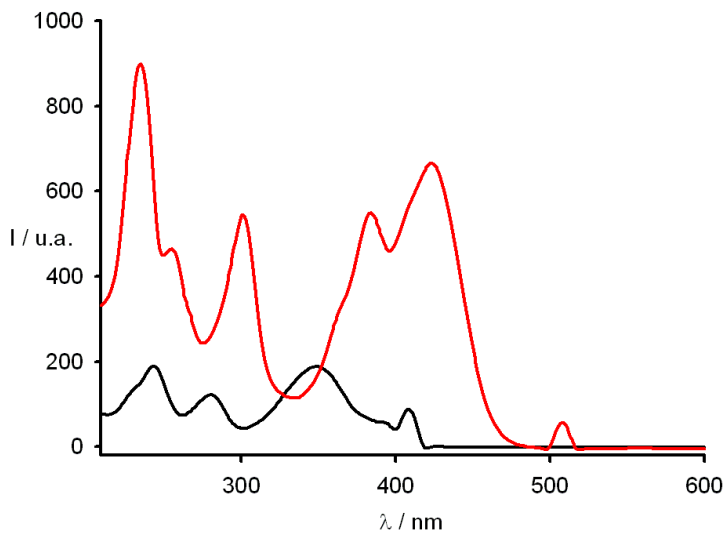

Figure SI 2. Excitation spectra of $1+1$ equiv. of $\mathrm{Hg}\left(\mathrm{ClO}_{4}\right)_{2}$ monitored at $418 \mathrm{~nm}$ (black) and $508 \mathrm{~nm}$ (red) in $\mathrm{CH}_{3} \mathrm{CN}$. 


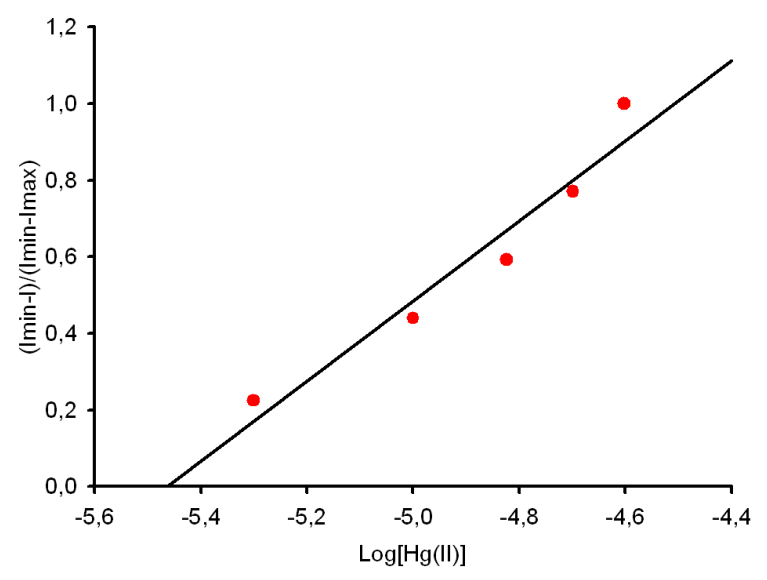

Figure SI 3. Fluorescence intensity of 1 at each concentration of $\mathbf{H g}^{2+}$ added, normalized between the minimum fluorescence intensity, found at zero equiv of $\mathrm{Hg}^{2+}$, and the maximum fluorescence intensity, found at $\left[\mathrm{Hg}^{2+}\right]=2.5 \times 10^{-5} \mathrm{M}$.

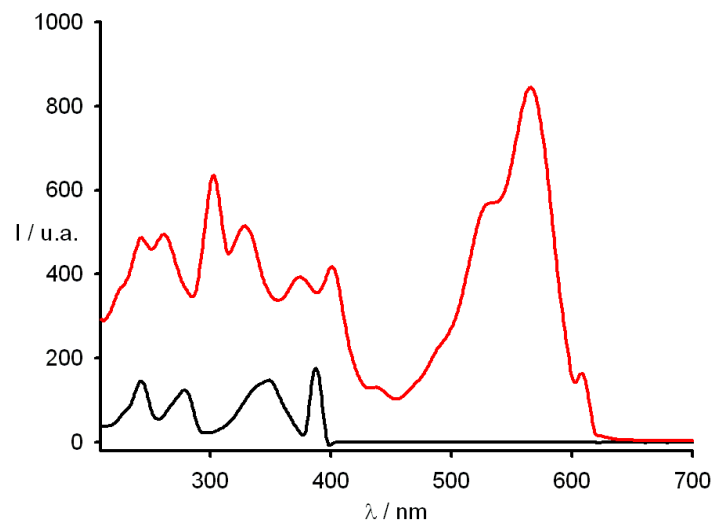

Figure SI 4. Excitation spectra of $\mathbf{1}+1$ equiv. of $\mathrm{Cu}(\mathrm{OTf})_{2}$ monitored at $388 \mathrm{~nm}$ (black) and $610 \mathrm{~nm}$ (red) in $\mathrm{CH}_{3} \mathrm{CN}$. 


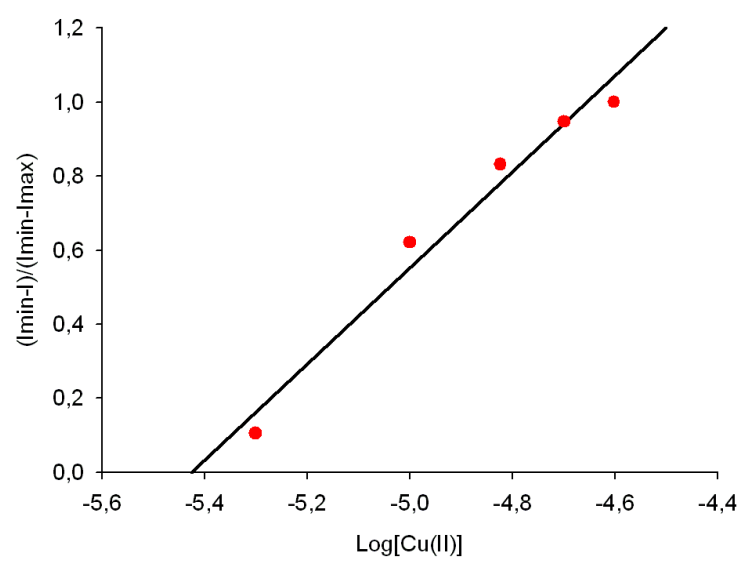

Figure SI 5. Fluorescence intensity of $\mathbf{1}$ at each concentration of $\mathrm{Cu}^{2+}$ added, normalized between the minimum fluorescence intensity, found at zero equiv of $\mathrm{Cu}^{2+}$, and the maximum fluorescence intensity, found at $\left[\mathrm{Cu}^{2+}\right]=2.5 \times 10^{-5} \mathrm{M}$. 

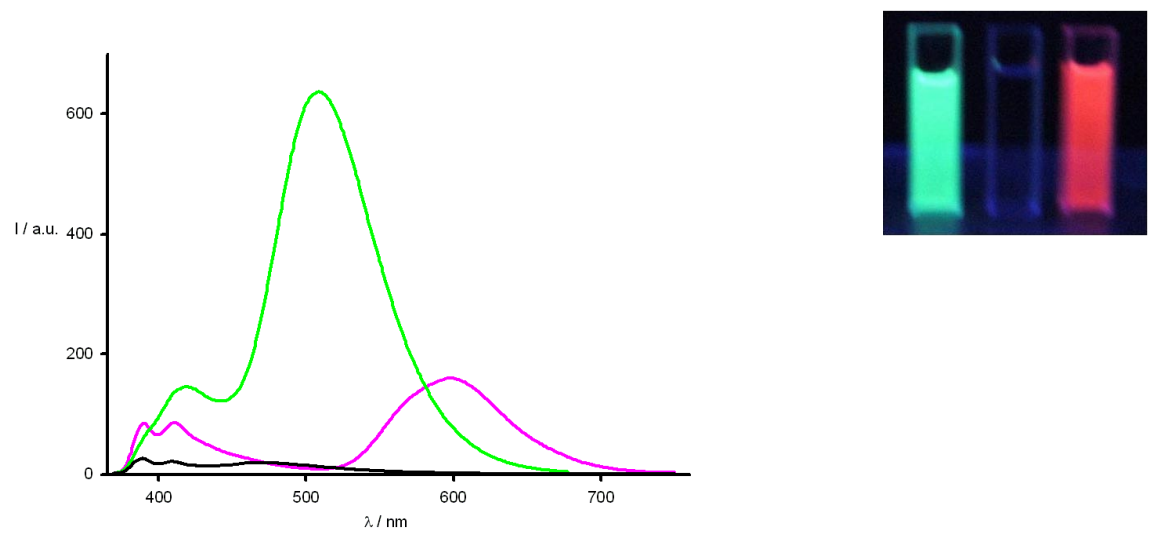

Figure SI 6. Comparison of the fluorescence spectra obtained after the addition of 1 equiv of $\mathrm{Hg}\left(\mathrm{ClO}_{4}\right)_{2}$ (green) or 1 equiv of $\mathrm{Cu}(\mathrm{OTf})_{2}$ (red) to a solution of $\mathbf{1}$ (c $=2.5 \times 10^{-5}$ mol dm ${ }^{-3}$ ) in $\mathrm{CH}_{3} \mathrm{CN}$. Black line represents the emission spectra of the free ligand in $\mathrm{CH}_{3} \mathrm{CN}$. Inset: Changes in the fluorescence shown by $\mathbf{1}$ (central) after addition of 1 equiv of $\mathrm{Hg}\left(\mathrm{ClO}_{4}\right)_{2}$ in $\mathrm{CH}_{3} \mathrm{CN}$ (left) or 1 equiv of $\mathrm{Cu}(\mathrm{OTf})_{2}$ in $\mathrm{CH}_{3} \mathrm{CN}$ (right). 


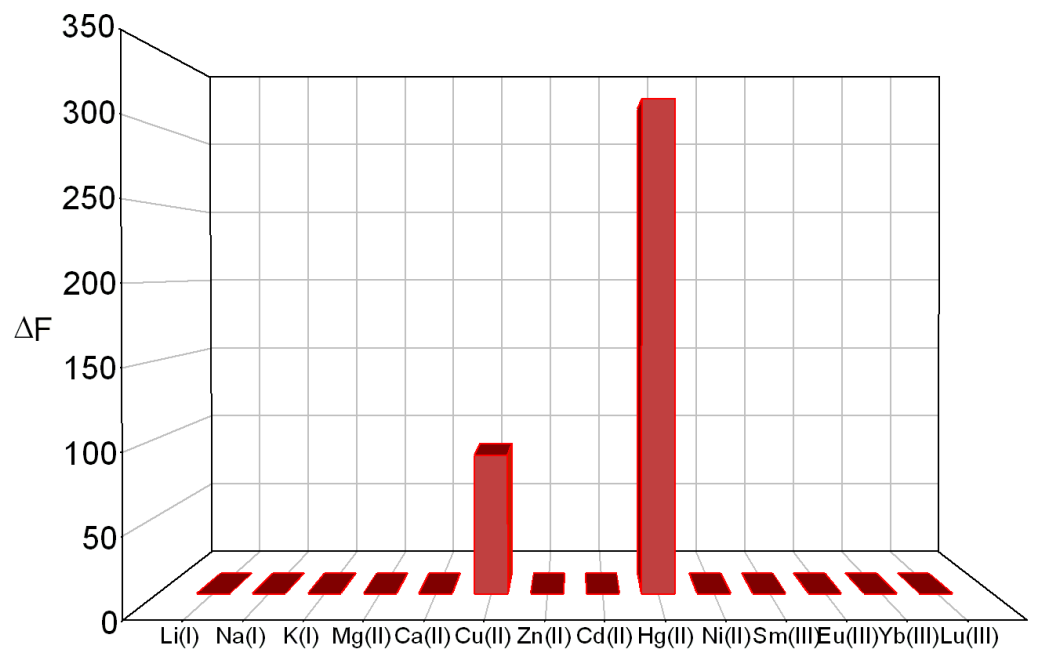

Figure SI 7. Fluorescence intensity increase, in $\mathrm{CH}_{3} \mathrm{CN}$, with respect to the free ligand 1, after addition of several metal ions. Excitation is at $\lambda=350 \mathrm{~nm}$ and emission is monitored at $\lambda=590 \mathrm{~nm}$ for $\mathrm{Cu}^{2+}$ and $\lambda=508 \mathrm{~nm}$ for $\mathrm{Hg}^{2+}$. 


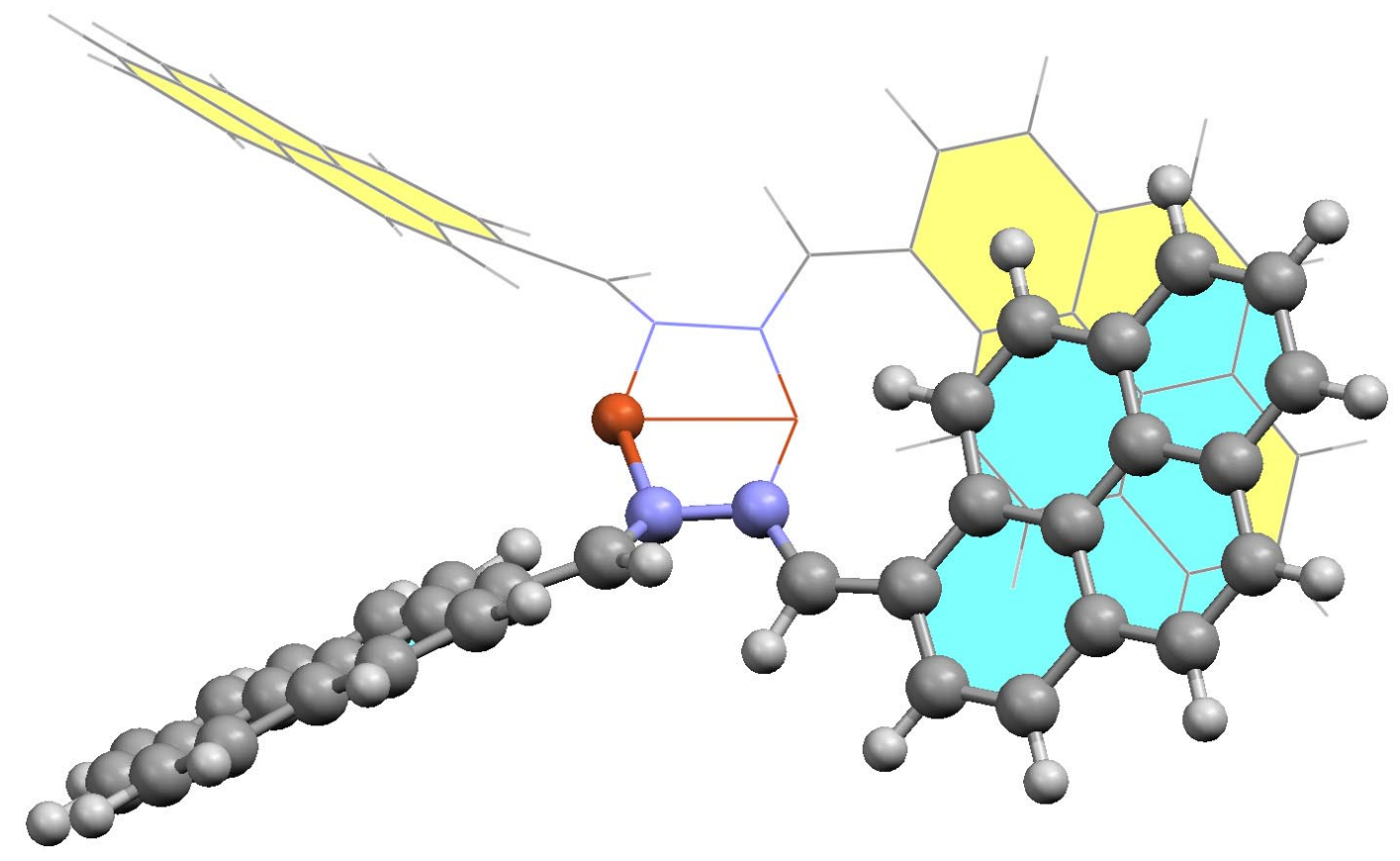

Figure SI 8. Calculated (B3LYP/6-31G*) structure for the $(\mathbf{1} \cdot \mathrm{Cu})_{2}{ }^{4+}$ complex. Only one half of the structure is highlighted by ball-and-stick representation. 


\section{Cartesian coordinates for the calculated structure of the $(1 \cdot \mathrm{Hg})_{2}{ }^{4+}$ complex (in $\AA$ ).-}

\begin{tabular}{|c|c|c|c|c|c|c|c|}
\hline $\mathrm{Hg}$ & 0.000000 & 0.000000 & 0.000000 & $\mathrm{H}$ & -3.392447 & 1.312422 & -3.992771 \\
\hline $\mathrm{Hg}$ & 4.371288 & 0.000000 & 0.000000 & C & 9.539114 & 0.633760 & -3.718089 \\
\hline $\mathrm{N}$ & 2.850937 & 2.069364 & 0.000000 & C & 9.539114 & -0.633760 & 3.718089 \\
\hline $\mathrm{N}$ & 2.850937 & -2.069364 & 0.000000 & C & -5.167826 & -0.122473 & 3.769726 \\
\hline $\mathrm{N}$ & 1.520351 & 2.027575 & 0.413771 & 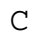 & -5.167826 & 0.122473 & -3.769726 \\
\hline $\mathrm{N}$ & 1.520351 & -2.027575 & -0.413771 & $\mathrm{H}$ & 10.168679 & 0.078747 & -4.405856 \\
\hline C & 3.556523 & 3.130815 & 0.329167 & $\mathrm{H}$ & 10.168679 & -0.078747 & 4.405856 \\
\hline C & 3.556523 & -3.130815 & -0.329167 & $\mathrm{I}$ & -5.797391 & -0.803798 & 4.33262 \\
\hline $\mathrm{C}$ & 0.814765 & 3.133408 & 0.303490 & $\mathrm{H}$ & -5.797391 & 0.803798 & -4.332629 \\
\hline $\mathrm{C}$ & 0.814765 & -3.133408 & -0.303490 & $\mathrm{C}$ & 10.084215 & 1.690576 & -2.974824 \\
\hline $\mathrm{H}$ & 3.089563 & 3.878572 & 0.976276 & C & 10.084215 & -1.690576 & 2.97482 \\
\hline $\mathrm{H}$ & 3.089563 & -3.878572 & -0.976276 & $\mathrm{C}$ & -5.712927 & 1.061618 & 3.252782 \\
\hline $\mathrm{H}$ & 1.281725 & 3.995455 & -0.181037 & $\mathrm{C}$ & -5.712927 & -1.061618 & -3.252782 \\
\hline $\mathrm{H}$ & 1.281725 & -3.995455 & 0.181037 & I & 11.135308 & 1.939613 & -3.095576 \\
\hline $\mathrm{C}$ & 4.927445 & 3.397114 & -0.019998 & $\mathrm{H}$ & 11.135308 & -1.939613 & 3.095576 \\
\hline $\mathrm{C}$ & 4.927445 & -3.397114 & 0.019998 & $\mathrm{H}$ & -6.764020 & 1.281481 & 3.420891 \\
\hline $\mathrm{C}$ & -0.556157 & 3.324514 & 0.698851 & I & -6.764020 & -1.281481 & -3.420891 \\
\hline C & -0.556157 & -3.324514 & -0.698851 & C & 9.303042 & 2.453999 & -2.086173 \\
\hline $\mathrm{C}$ & 5.156695 & 1.548626 & -1.738482 & $\mathrm{C}$ & 9.303042 & -2.453999 & 2.086173 \\
\hline $\mathrm{C}$ & 5.156695 & -1.548626 & 1.738482 & $\mathrm{C}$ & -4.931754 & 1.987310 & 2.534724 \\
\hline C & -0.785407 & 1.169742 & 2.013024 & C & -4.931754 & -1.987310 & -2.53472 \\
\hline $\mathrm{C}$ & -0.785407 & -1.169742 & -2.013024 & 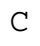 & 9.856922 & 3.554138 & -1.351018 \\
\hline $\mathrm{H}$ & 4.102341 & 1.607901 & -2.002048 & $\mathrm{C}$ & 9.856922 & -3.554138 & 1.351018 \\
\hline $\mathrm{H}$ & 4.102341 & -1.607901 & 2.002048 & C & -5.485634 & 3.212229 & 2.034388 \\
\hline $\mathrm{H}$ & 0.268947 & 1.175120 & 2.283119 & C & -5.485634 & -3.212229 & -2.03438 \\
\hline $\mathrm{H}$ & 0.268947 & -1.175120 & -2.283119 & $\mathrm{H}$ & 10.908334 & 3.793663 & -1.48127 \\
\hline $\mathrm{C}$ & 5.712192 & 2.620522 & -0.921820 & $\dot{H}$ & 10.908334 & -3.793663 & 1.48127 \\
\hline C & 5.712192 & -2.620522 & 0.921820 & ¥ & -6.537046 & 3.420872 & 2.209904 \\
\hline C & -1.340904 & 2.383284 & 1.427180 & 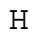 & -6.537046 & -3.420872 & -2.209904 \\
\hline $\mathrm{C}$ & -1.340904 & -2.383284 & -1.427180 & C & 9.077937 & 4.302439 & -0.514807 \\
\hline C & 7.103417 & 2.909836 & -1.058662 & C & 9.077937 & -4.302439 & 0.514807 \\
\hline $\mathrm{C}$ & 7.103417 & -2.909836 & 1.058662 & & -4.706649 & 4.112619 & 1.364688 \\
\hline $\mathrm{C}$ & -2.732129 & 2.639394 & 1.619108 & $\mathrm{C}$ & -4.706649 & -4.112619 & -1.364688 \\
\hline $\mathrm{C}$ & -2.732129 & -2.639394 & -1.619108 & I & 9.503366 & 5.142091 & 0.026400 \\
\hline C & 7.923465 & 2.136121 & -1.933153 & I & 9.503366 & -5.142091 & -0.026400 \\
\hline C & 7.923465 & -2.136121 & 1.933153 & $\mathrm{H}$ & -5.132078 & 5.043530 & 1.002299 \\
\hline $\mathrm{C}$ & -3.552177 & 1.706448 & 2.321234 & & -5.132078 & -5.043530 & -1.002299 \\
\hline $\mathrm{C}$ & -3.552177 & -1.706448 & -2.321234 & C & 7.689581 & 4.011446 & -0.34582 \\
\hline C & 7.369340 & 1.051758 & -2.696735 & C & 7.689581 & -4.011446 & 0.345820 \\
\hline C & 7.369340 & -1.051758 & 2.696735 & $\mathrm{C}$ & -3.318293 & 3.861291 & 1.140929 \\
\hline C & -2.998052 & 0.491304 & 2.852577 & $\mathrm{C}$ & -3.318293 & -3.861291 & -1.14092 \\
\hline C & -2.998052 & -0.491304 & -2.852577 & 0 & 6.869065 & 4.794759 & 0.49455 \\
\hline C & 5.988020 & 0.811571 & -2.607521 & C & 6.869065 & -4.794759 & -0.49455 \\
\hline C & 5.988020 & -0.811571 & 2.607521 & & -2.497777 & 4.796820 & 0.47414 \\
\hline C & -1.616732 & 0.273806 & 2.717138 & C & -2.497777 & -4.796820 & -0.47414 \\
\hline C & -1.616732 & -0.273806 & -2.717138 & $\mathrm{H}$ & 7.300062 & 5.642365 & 1.018588 \\
\hline $\mathrm{H}$ & 5.535088 & 0.083075 & -3.276522 & $\mathrm{H}$ & 7.300062 & -5.642365 & -1.01858 \\
\hline $\mathrm{H}$ & 5.535088 & -0.083075 & 3.276522 & $\mathrm{H}$ & -2.928774 & 5.732090 & 0.13017 \\
\hline $\mathrm{H}$ & -1.163800 & -0.573746 & 3.226966 & $\mathrm{H}$ & -2.928774 & -5.732090 & -0.13017 \\
\hline $\mathrm{H}$ & -1.163800 & 0.573746 & -3.226966 & $\mathrm{C}$ & 5.534430 & 4.491531 & 0.65323 \\
\hline C & 8.197718 & 0.316262 & -3.586007 & $\mathrm{C}$ & 5.534430 & -4.491531 & -0.65323 \\
\hline C & 8.197718 & -0.316262 & 3.586007 & $\mathrm{C}$ & -1.163142 & 4.531444 & 0.25803 \\
\hline $\mathrm{C}$ & -3.826430 & -0.407150 & 3.576827 & $\mathrm{C}$ & -1.163142 & -4.531444 & -0.25803 \\
\hline $\mathrm{C}$ & -3.826430 & 0.407150 & -3.576827 & $\mathrm{H}$ & 4.927719 & 5.100971 & 1.31767 \\
\hline $\mathrm{H}$ & 7.763735 & -0.487561 & -4.174561 & $\mathrm{H}$ & 4.927719 & -5.100971 & -1.31767 \\
\hline $\mathrm{H}$ & 7.763735 & 0.487561 & 4.174561 & $\mathrm{H}$ & -0.556431 & 5.261433 & -0.27112 \\
\hline $\mathrm{H}$ & -3.392447 & -1.312422 & 3.992771 & $\mathrm{H}$ & -0.556431 & -5.261433 & 0.27112 \\
\hline
\end{tabular}




\section{Cartesian coordinates for the calculated structure of the $(1 \cdot \mathrm{Cu})_{2}{ }^{4+}$ complex (in $\AA$ ).-}

\begin{tabular}{|c|c|c|c|c|c|c|c|}
\hline $\mathrm{Cu}$ & 0.000000 & 0.000000 & 0.000000 & $\mathrm{H}$ & -4.923581 & -0.246837 & -4.497460 \\
\hline $\mathrm{Cu}$ & 2.363446 & 0.000000 & 0.000000 & $\mathrm{C}$ & 8.676263 & 2.129671 & -4.195798 \\
\hline $\mathrm{N}$ & 1.879252 & 1.821677 & 0.000000 & C & 8.676263 & -2.129671 & 4.195798 \\
\hline & 1.879252 & -1.821677 & 0.000000 & C & -6.312817 & 1.875650 & 4.315340 \\
\hline $\mathrm{N}$ & 0.484194 & 1.818434 & 0.108642 & $\mathrm{C}$ & -6.312817 & -1.875650 & -4.315340 \\
\hline & 0.484194 & -1.818434 & -0.108642 & $\mathrm{H}$ & 9.353656 & 1.788722 & -4.971649 \\
\hline & 2.521818 & 2.947453 & 0.318100 & $\mathrm{H}$ & 9.353656 & -1.788722 & 4.971649 \\
\hline C & 2.521818 & -2.947453 & -0.318100 & $\mathrm{H}$ & -6.990210 & 1.489038 & 5.069476 \\
\hline C & -0.158372 & 2.961178 & -0.141753 & $\mathrm{H}$ & -6.990210 & -1.489038 & -5.069476 \\
\hline C & -0.158372 & -2.961178 & 0.141753 & C & 8.968205 & 3.291554 & -3.476304 \\
\hline $\mathrm{H}$ & 1.931234 & 3.667466 & 0.886215 & C & 8.968205 & -3.291554 & 3.476304 \\
\hline $\mathrm{H}$ & 1.931234 & -3.667466 & -0.886215 & C & -6.604759 & 3.078374 & 3.666419 \\
\hline $\mathrm{H}$ & 0.432212 & 3.713791 & -0.665916 & C & -6.604759 & -3.078374 & -3.666419 \\
\hline $\mathrm{H}$ & 0.432212 & -3.713791 & 0.665916 & $\mathrm{H}$ & 9.875186 & 3.848374 & -3.695585 \\
\hline C & 3.855035 & 3.362450 & 0.049386 & $\mathrm{H}$ & 9.875186 & -3.848374 & 3.695585 \\
\hline $\mathrm{C}$ & 3.855035 & -3.362450 & -0.049386 & $\mathrm{H}$ & -7.511734 & 3.621126 & 3.918517 \\
\hline C & -1.491589 & 3.359411 & 0.151233 & $\mathrm{H}$ & -7.511734 & -3.621126 & -3.918517 \\
\hline C & -1.491589 & -3.359411 & -0.151233 & $\mathrm{C}$ & 8.100258 & 3.764620 & -2.465484 \\
\hline C & 4.552307 & 1.600220 & -1.636544 & C & 8.100258 & -3.764620 & 2.465484 \\
\hline $\mathrm{C}$ & 4.552307 & -1.600220 & 1.636544 & $\mathrm{C}$ & -5.736812 & 3.610882 & 2.685611 \\
\hline C & -2.188861 & 1.499771 & 1.729065 & $\mathrm{C}$ & -5.736812 & -3.610882 & -2.685611 \\
\hline C & -2.188861 & -1.499771 & -1.729065 & C & 8.388968 & 4.948465 & -1.733641 \\
\hline $\mathrm{H}$ & 3.665295 & 1.016480 & -1.422259 & C & 8.388968 & -4.948465 & 1.733641 \\
\hline $\mathrm{H}$ & 3.665295 & -1.016480 & 1.422259 & C & -6.025522 & 4.836266 & 2.025673 \\
\hline $\mathrm{H}$ & -1.301849 & 0.929850 & 1.480349 & $\mathrm{C}$ & -6.025522 & -4.836266 & -2.025673 \\
\hline $\mathrm{H}$ & -1.301849 & -0.929850 & -1.480349 & $\mathrm{H}$ & 9.301136 & 5.497195 & -1.950784 \\
\hline C & 4.789126 & 2.798035 & -0.911412 & $\mathrm{H}$ & 9.301136 & -5.497195 & 1.950784 \\
\hline C & 4.789126 & -2.798035 & 0.911412 & $\mathrm{H}$ & -6.937690 & 5.371069 & 2.275155 \\
\hline C & -2.425680 & 2.738700 & 1.076659 & $\mathrm{H}$ & -6.937690 & -5.371069 & -2.275155 \\
\hline C & -2.425680 & -2.738700 & -1.076659 & C & 7.524123 & 5.406670 & -0.760137 \\
\hline $\mathrm{C}$ & 6.000079 & 3.511006 & -1.181376 & C & 7.524123 & -5.406670 & 0.760137 \\
\hline C & 6.000079 & -3.511006 & 1.181376 & $\mathrm{C}$ & -5.160677 & 5.351713 & 1.081229 \\
\hline C & -3.636633 & 3.434301 & 1.388664 & C & -5.160677 & -5.351713 & -1.081229 \\
\hline C & -3.636633 & -3.434301 & -1.388664 & $\mathrm{H}$ & 7.754872 & 6.314761 & -0.211474 \\
\hline C & 6.902867 & 3.041316 & -2.177163 & $\mathrm{H}$ & 7.754872 & -6.314761 & 0.211474 \\
\hline $\mathrm{C}$ & 6.902867 & -3.041316 & 2.177163 & $\mathrm{H}$ & -5.391426 & 6.290910 & 0.587699 \\
\hline C & -4.539421 & 2.906060 & 2.354667 & $\mathrm{H}$ & -5.391426 & -6.290910 & -0.587699 \\
\hline $\mathrm{C}$ & -4.539421 & -2.906060 & -2.354667 & C & 6.322544 & 4.714278 & -0.471125 \\
\hline C & 6.616568 & 1.855228 & -2.908332 & C & 6.322544 & -4.714278 & 0.471125 \\
\hline C & 6.616568 & -1.855228 & 2.908332 & C & -3.959098 & 4.677790 & 0.751438 \\
\hline $\mathrm{C}$ & -4.253122 & 1.678478 & 3.013797 & $\mathrm{C}$ & -3.959098 & -4.677790 & -0.751438 \\
\hline C & -4.253122 & -1.678478 & -3.013797 & C & 5.412295 & 5.203990 & 0.505074 \\
\hline C & 5.429579 & 1.146383 & -2.599752 & C & 5.412295 & -5.203990 & -0.505074 \\
\hline C & 5.429579 & -1.146383 & 2.599752 & $\mathrm{C}$ & -3.048849 & 5.224849 & -0.193818 \\
\hline C & -3.066133 & 0.989298 & 2.663493 & $\mathrm{C}$ & -3.048849 & -5.224849 & 0.193818 \\
\hline $\mathrm{C}$ & -3.066133 & -0.989298 & -2.663493 & $\mathrm{H}$ & 5.655657 & 6.111787 & 1.048711 \\
\hline $\mathrm{H}$ & 5.206199 & 0.232330 & -3.143271 & $\mathrm{H}$ & 5.655657 & -6.111787 & -1.048711 \\
\hline $\mathrm{H}$ & 5.206199 & -0.232330 & 3.143271 & $\mathrm{H}$ & -3.292211 & 6.163451 & -0.682348 \\
\hline $\mathrm{H}$ & -2.842753 & 0.044457 & 3.151532 & $\mathrm{H}$ & -3.292211 & -6.163451 & 0.682348 \\
\hline $\mathrm{H}$ & -2.842753 & -0.044457 & -3.151532 & $\mathrm{C}$ & 4.223588 & 4.560984 & 0.734547 \\
\hline $\mathrm{C}$ & 7.517591 & 1.417440 & -3.915765 & C & 4.223588 & -4.560984 & -0.73454 \\
\hline C & 7.517591 & -1.417440 & 3.915765 & $\mathrm{C}$ & -1.860142 & 4.596673 & -0.46123 \\
\hline C & -5.154145 & 1.181387 & 3.993329 & C & -1.860142 & -4.596673 & 0.46123 \\
\hline C & -5.154145 & -1.181387 & -3.993329 & $\mathrm{H}$ & 3.528758 & 4.982064 & 1.45539 \\
\hline $\mathrm{H}$ & 7.287027 & 0.514618 & -4.474734 & $\mathrm{H}$ & 3.528758 & -4.982064 & -1.455391 \\
\hline $\mathrm{H}$ & 7.287027 & -0.514618 & 4.474734 & $\mathrm{H}$ & -1.165312 & 5.059993 & -1.155679 \\
\hline $\mathrm{H}$ & -4.923581 & 0.246837 & 4.497460 & $\mathrm{H}$ & -1.165312 & -5.059993 & 1.15567 \\
\hline
\end{tabular}




\section{References.-}

1.- (a) Dawson W.R., Windsor, M.W., J. Phys. Chem. 1968, 72, 3251. (b) Melhuish,

W.H., J. Phys. Chem. 1961, 65, 229.

2.- Valeur B. Molecular Fluorescence: Principles and Applications, Wiley-VCH, 2001, p 161.

3.- CAChe WorkSystem Pro 6.1.12, Fujitsu Limited 2000-2004.

4.- See for instance: Linnanto, J.; Korppi-Tommola, J. J. Comput. Chem., 2004, 25, 123-138. Bañuelos Prieto, J.; López Arbeloa, F.; Martínez Martínez, V.; Arbeloa López, T.; López Arbeloa, I. Phys. Chem. Chem. Phys., 2004, 6, 4247-4253.

5.- Gaussian 03, Revision B.03, Frisch, M. J.; Trucks, G. W.; Schlegel, H. B.; Scuseria, G. E.; Robb, M. A.; Cheeseman, J. R.; Montgomery, Jr., J. A.; Vreven, T.; Kudin, K. N.; Burant, J. C.; Millam, J. M.; Iyengar, S. S.; Tomasi, J.; Barone, V.; Mennucci, B.; Cossi, M.; Scalmani, G.; Rega, N.; Petersson, G. A.; Nakatsuji, H.; Hada, M.; Ehara, M.; Toyota, K.; Fukuda, R.; Hasegawa, J.; Ishida, M.; Nakajima, T.; Honda, Y.; Kitao, O.; Nakai, H.; Klene, M.; Li, X.; Knox, J. E.; Hratchian, H. P.; Cross, J. B.; Bakken, V.; Adamo, C.; Jaramillo, J.; Gomperts, R.; Stratmann, R. E.; Yazyev, O.; Austin, A. J.; Cammi, R.; Pomelli, C.; Ochterski, J. W.; Ayala, P. Y.;

Morokuma, K.; Voth, G. A.; Salvador, P.; Dannenberg, J. J.; Zakrzewski, V. G.; Dapprich, S.; Daniels, A. D.; Strain, M. C.; Farkas, O.; Malick, D. K.; Rabuck, A. D.; Raghavachari, K.; Foresman, J. B.; Ortiz, J. V.; Cui, Q.; Baboul, A. G.; Clifford, S.; Cioslowski, J.; Stefanov, B. B.; Liu, G.; Liashenko, A.; Piskorz, P.; Komaromi, I.; Martin, R. L.; Fox, D. J.; Keith, T.; Al-Laham, M. A.; Peng, C. Y.; Nanayakkara, A.; Challacombe, M.; Gill, P. M. W.; Johnson, B.; Chen, W.; Wong, M. W.; Gonzalez, C.; and Pople, J. A.; Gaussian, Inc., Wallingford CT, 2004.

6.- Bartolottiand, L.J.; Fluchick, K. In Reviews in Computational Chemistry, Lipkowitz. K.B;. Boyd, B.D., Eds.; VCH, New York, 1996; Vol. 7, pp. 187-216.

7.- Obtained from the Extensible Computational Chemistry Environment Basis Set Database, Version 02/25/04, as developed and distributed by the Molecular Science Computing Facility, Environmental and Molecular Sciences Laboratory which is part of the Pacific Northwest Laboratory, P.O. Box 999, Richland, Washington 99352, USA, and funded by the U.S. Department of Energy. The Pacific Northwest Laboratory is a multi-program laboratory operated by Battelle Memorial Institute for the U.S. Department of Energy under contract DE-AC06-76RLO 1830.

8.- Wiberg, K. Tetrahedron, 1968, 24, 1083. 